\title{
Diffraktive Elemente für die Lasermaterialbearbeitung
}

\author{
M. Pahlke, C. Haupt, H.-J. Tiziani
}

Universität Stuttgart, Institut für Technische Optik, Pfaffenwaldring 9, D - 7000 Stuttgart 80

\section{Einleitung}

In der Materialbearbeitung werden immer häufiger $\mathrm{CO}_{2}$-Hochleistungslaser eingesetzt. Beim Schneiden, Schweißen und Bohren sind Energiedichten von $\sim 10^{7} \mathrm{~W} / \mathrm{cm}^{2}$ erforderlich, die durch Fokussierung des Laserstrahls erreicht werden, wobei als optische Elemente Linsen bzw. Hohlspiegel eingesetzt werden.

Geringere Energiedichten sind zum Härten erforderlich. Dabei muß die Temperatur einer zu härtenden Fläche für eine bestimmte Zeit innerhalb eines bestimmten Temperaturintervalls liegen. In der Praxis wird ein Laserstrahl über die zu härtende Fläche geführt. Ein Aufschmelzen der Oberfläche muß dabei vermieden werden. Die so erzeugte Intensitätsverteilung auf dem Werkstück führt nicht zu idealen Ergebnissen, weil das Temperaturfeld im Werkstück den Härtungsprozeß nicht im erforderlichen Maß unterstützt. Ein optimiertes Intensitätsprofil zum Härten wurde von Burger /1/vorgeschlagen. Dieses Intensitätsprofil kann mit klassischen optischen Komponenten nicht erzeugt werden. Diffraktive Elemente (DE) können grundsătzlich derartige Intensitätsverteilungen erzeugen, deren Struktur jedoch nur mit aufwendigen numerischen Verfahren berechnet werden können.

Das Härten von runden Öffnungen, beispielsweise bei Ventilen oder die Lauffläche in einem Konuslager, könnte dadurch erleichtert werden, daß die gesamte zu hărtende Flache mit einer ringförmigen Intensitătsverteilung bearbeitet wird. Die Erzeugung dieser Intensitätsverteilung soll hier beschrieben werden. Dazu wird ein analytisches Verfahren zur Berechnung der Beugungsstrukturen und der lithographische Herstellungsproze $B$ eines $\mathrm{DE}$ beschrieben. Ein diffraktives Element wurde hergestellt und untersucht.

DE zur Materialbearbeitung werden in Form von computergenerierten Phasenhologrammen realisiert. Sie sollen einen möglichst hohen Beugungswirkungsgrad und eine möglichst geringe Absorption besitzen. Die Funktion der Elemente beruht auf der Beugung von Lichtwellen an einer Oberflächenstruktur auf einem ebenen Substrat. Für den Einsatz mit Hochleistungslasern bieten diffraktive Reflexionselemente die gleichen Vorteile wie konventionelle Reflexionsoptiken, insbesondere im Hinblick auf Absorption und Kühlung.

\section{Berechnung der Oberflächenstruktur diffraktiver Reflexionsoptiken}

Die Oberflächenstruktur wird mit der skalaren Beugungstheorie berechnet. Das Beugungsintegral beschreibt das komplexe Lichtfeld in einer Ebene B, das von einer Apertur in Ebene A erzeugt wird (Fig. 1). In Ebene A liegt die erwünschte Intensitätsverteilung. Das Beugungsintegral wird in der FraunhoferNäherung verwendet (Gleichung 1).

$$
h\left(x^{\prime}, y^{\prime}\right) \propto \int_{-\infty}^{\infty} a(x, y) e^{-i \frac{2 \pi\left(x x^{\prime}+y y^{\prime}\right)}{\lambda z}} d x d y
$$


Das hat in der Praxis zur Folge, daß zur Rekonstruktion ein zusätzliches fokussierendes Element benötigt

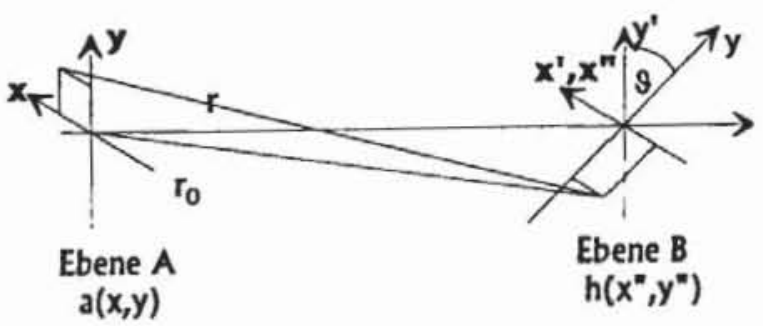

Fig. 1: Die geometrischen Verhaltnisse zur Berechnung von DEs; Ebene A: Rekonstruktionsebene; Ebene B: Ebene des DE wird, wodurch die Beugungsstrukturen gröber werden und damit leichter herstellbar sind. Für die Berechnung bedeutet dies, daß das komplexe Lichtfeld der Apertur $\mathrm{A}$ in der Ebene B mit einer Fouriertransformation ermittelt werden kann. Die Fraunhofer-Näherung ist jedoch beschränkt auf den Fall, daß die Apertur A und die Ebene B parallel zueinander sind und die Lichtwelle senkrecht zur Apertur einfällt.

Die diffraktiven Reflexionselementen werden jedoch schräg beleuchtet, was zur Folge hat, daß die Fraunhofer-Näherung erweitert werden muß $/ 2 /, 3 /$. Berücksichtigt man die geometrischen Bedingungen des Strahlengangs, so kann das Beugungsintegral derart angenähert werden, da $\beta$ der schräge Einfall des Laserstrahls auf das diffraktive Element berücksichtigt wird. Man erhălt als Ergebnis

$$
h\left(x^{n}, y^{n}\right) \propto \int_{-\infty}^{\infty} a(x, y) e^{-i \frac{2 x\left(x x^{*}+y y^{n} \cos 9\right)}{\lambda_{0}}} d x d y
$$

mit der Größe $\mathbf{r}_{0}$

$$
r_{0}=\sqrt{z^{2}+x^{\prime 2}+y^{\prime 2}+2 z y^{n} \sin \vartheta}
$$

Diese modifizierte Näherung des Beugungsintegrals führt dazu, daß die Beugungsstrukturen des DE in y-Richtung mit dem Faktor $1 / \cos \vartheta$ gestreckt werden. Im Vergleich zur Fraunhofer-Năherung werden die Raumfrequenzen für $\psi<0$ größer und für die $\psi>0$ kleiner.

Dieses Ergebnis dient als Grundlage für das folgende Berechnungsverfahren, das sich insbesondere zur

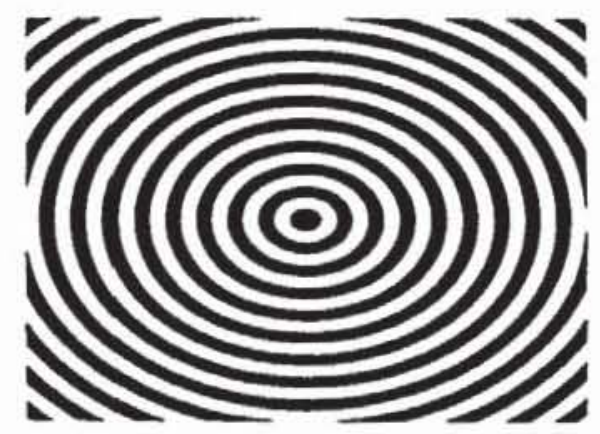

Fig. 2: Beugungsstruktur fulr Kreisring Berechnung von diffraktiven Strukturen zur Erzeugung von Fokuskurven eignet $/ 4 /, / 5 /$. Die gewunschte Fokuskurve soll sich durch eine Funktion $y=f(x)$ beschreiben lassen. Die Intensitätsverteilung $\mathrm{a}(\mathrm{x}, \mathrm{y})$ wird dann mit Hilfe der Dirac'schen Deltafunktion dargestellt, und man erhält $a(x, y)=\delta(y-f(x))$. Setzt man $a(x, y)$ in Gleichung (2) ein, dann läßt sie sich über eine Variable direkt integrieren. Zur Lősung des verbleibenden Integrals wird die Methode der Stationăren Phase verwendet. $\mathrm{Da}$ die meiste Information über eine Wellenfront in der Phasenfunktion /6/ enthalten ist, wird nur diese von dem berechneten komplexen Feld zur Erzeugung der Beugungsstruktur verwendet. Mit der Berücksichtigung der reellen Amplitude wären zusätzliche Energieverluste verbunden.

Die Intensitătsverteilung eines Kreisrings mit dem Radius $\rho$ ist gegeben mit

$$
a(x, y)=\delta\left(y-\sqrt{\rho^{2}-x^{2}}\right)
$$


Die daraus resultierende Phasenfunktion lautet

$$
\Phi(\xi, \psi)=\frac{2 \pi \rho}{\lambda r_{0}} \sqrt{\xi^{2}+\psi^{2} \cos ^{2} \vartheta}
$$

\section{Herstellung der Diffraktiven Elemente}

Aus herstellungstechnischen Gründen ist es notwendig den Wertebereich der Phasenfunktion auf einen diskreten, endlichen Wertebereich zu begrenzen (quantisieren). Die Phasenfunktion kann nun auf Grund der Periodizität trigonometrischer Funktionen in den Wertebereich zwischen Null und $2 \pi$ abgetragen und in äquidistante Intervalle unterteilt werden. Die Werte eines Intervalls werden schließlich auf einen Wert festgelegt, was einer Annäherung der berechneten Funktion durch eine Treppenfunktion entspricht. Die Effizienz eines diffraktiven Elements steigt mit der Anzahl der Stufen, da die berechnete Funktion mit zunehmender Zahl der Stufen immer besser angenähert wird. Begrenzt man den Wertebereich der obigen Phasenfunktion auf zwei Werte, dann erhält man die Oberflächenstruktur in Fig. 2. Bei einem Einfallswinkel $\vartheta=45^{\circ}$ und einer Wellenlänge von $10.6 \mu \mathrm{m}$ beträgt die Tiefe der Struktur $3,75 \mu \mathrm{m}$.

Als Substratmaterial der diffraktiven Strukturen wurde Silizium ausgewählt $/ 7 /$, weil es im Hinblick auf die thermischen und verfahrenstechnischen Eigenschaften für die Anwendung geeignet ist. Darüber hinaus sind die erforderlichen Prozeßparameter für Silizium bei der Strukturierung mit reaktivem Ionenstrahlätzen aus der Mikroelektronik bekannt.

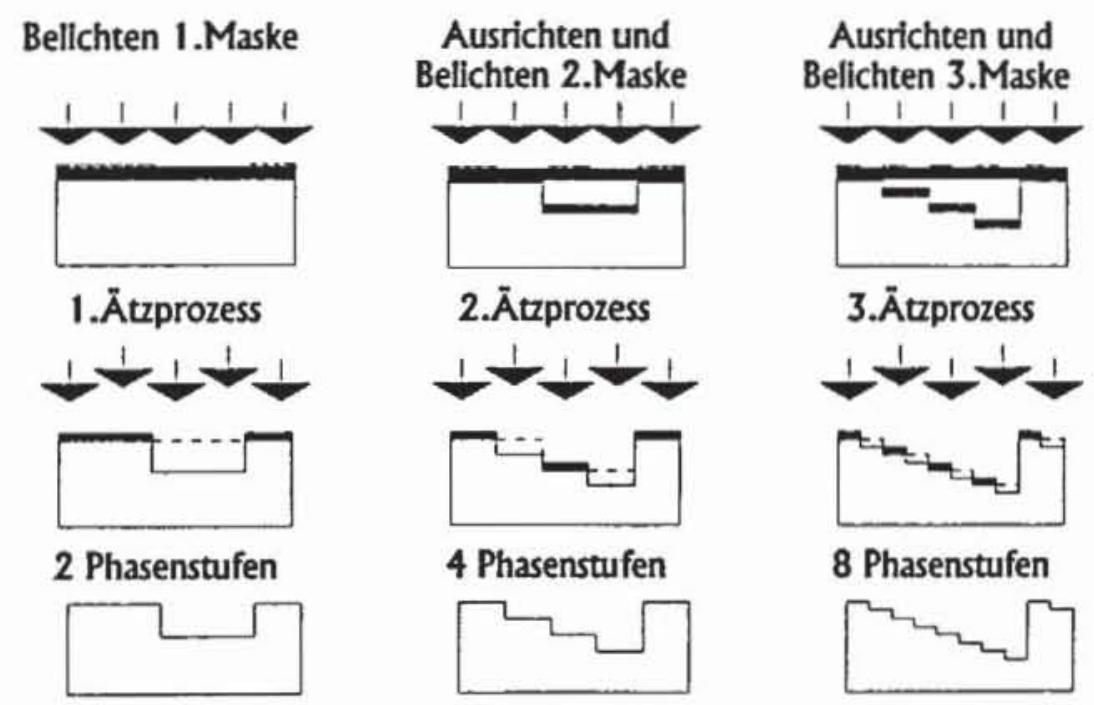

Fig. 3: dreistufiger Åtzprozeß zur Erzeugung einer 8-Stufigen Beugungsstruktur

Im ersten Schritt werden mit einem Laserscanner die berechneten Muster in den Photoresist von Chrommaskenblanks geschrieben. Die Anzahl der Stufen wächst als Zweierpotenz mit der Anzahl der eingesetzten Masken. Dazu wird im ersten Schritt die erste Maskenstruktur photolithographisch auf das Substratmaterial durch Ätzung übertragen, wobei die Strukturen eine bestimmte Tiefe bekommen. Danach wird die zweite Maske zu der bereits geătzten Struktur positioniert und wieder photolithographisch übertragen. Durch den zweiten Ätzprozeß erhält man schließlich vier Stufen. In Fig. 3 ist der Prozeß schematisch dargestellt. Da Silizium für elektromagnetische Strahlung der Wellenlänge $\lambda=10,6 \mu \mathrm{m}$ transparent ist muß nach der Strukturierung eine harte, hochreflektierende Schicht aufgebracht werden. 


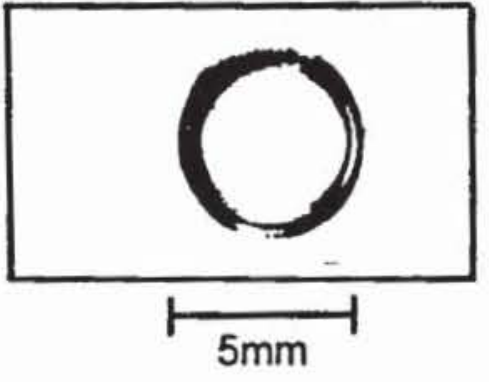

Fig. 4: Optische Rekonstruktion der Beugungsstruktur in Fig. 2

Es wurde ein diffraktives Element mit vier Stufen hergestellt. Als reflektierende Schicht wurde Gold aufgedampft. Die optische Rekonstruktion zeigt, daß beim Ätzprozeß die notwendige Tiefe von $5,6 \mu \mathrm{m}$ der Struktur mit großer Genauigkeit hergestellt wurde, was sich dadurch außert, daß der Gleichlichtanteil verschwindet. Der rekonstruierte Kreisring (Fig. 4) weist keine meßbaren Abweichungen von der Kreisform auf. Die Beugungseffizienz beträgt $73 \%$ bei der Rekonstruktion mit einem $\mathrm{CO}_{2}$-Laborlaser (Ausgangsleistung: 20W). Die Erwärmung eines ungekühlten diffraktiven Elements war dabei gering. Im Hochleistungslaserstrahl wurde das ungekühlte Element mehrmals kurzzeitig mit 1kW Strahlleistung belastet, ohne daß eine Beschädigung der Beugungsstrukturen auftrat.

\section{Zusammenfassung und Ausblick}

Es wird ein Verfahren zur Berechnung von schrăg beleuchteten Beugungsstrukturen vorgestellt. Das Verfahren ist furr die Erzeugung von Fokuskurven geeignet. Die berechnete Beugungsstruktur wurde mit einem Mehrmaskenprozess durch reaktives Ionenstrahlätzen in Silizium hergestellt. Mit einem vierstufigen diffraktiven Element wird eine Effizienz von 73\% erreicht. Die erforderlichen Tiefen der Beugungs-

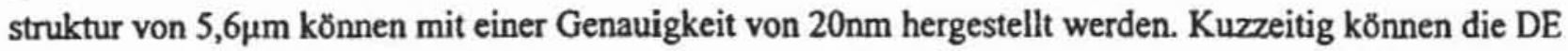
mit einer Strahlleistung von $1 \mathrm{~kW}$ belastet werden. Die Strahlungsfestigkeit läßt sich durch die Kühlung der DE erhőhen. Zur Steigerung der Beugungseffizienz der DE muß die Anzahl der Quantisierungsstufen erhőht werden.

\section{Danksagung}

Die Autoren danken dem "Hahn-Schickard Institut für Mikro- und Informationstechnik" in VillingenSchwenningen für die Silizium Lithographie, und dem "Institut für Strahlwerkzeuge" an der Universităt Stuttgart für die Strahlzeit an einem Hochleistungslaser. Der besonderer Dank gilt der "Deutschen Forschungsgemeinschaft" für die finanzielle Unterstützung der Arbeiten im Rahmen des Sonderforschungsbereichs 349.

\section{Literatur}

II/ Burger, D., Optimierung der Strahlqualităt beim Laserhărten, in LASER Optoelektronik in der Technik, Springer-Verlag Berlin, 1990.

12/ Patorski, K., Fraunhofer diffraction patterns of tilted planar objects, Optica Acta 30, 673 (1982).

B/ Leseberg, D.,Frere, C., Computer-generated holograms of 3D-Objects composed of tilted planar segment, Appl. Opt., 27(14), 3020 (1988).

14/ Jaroszewicz, Z., Kolodziejczyk, A., Mouriz, D., Bara, S., Analytic design of computer-generated Fourier-transform holograms for plane curves reconstruction, J. Opt. Soc. Am. A, 8 ,559 (1991).

15/ Pahlke, M.; Optimienung von Beugungsstrukturen fur hohen Beugungswirkungsgrad, Diplomarbeit Universităt Stuttgart, Institut filr Technische Optik, (1992).

16/ Kermisch, D., Immage Reconstruction from Phase Information Only, J. Opt. Soc. Am., 60, 15(1970).

7/ Haupt, C., Pahlke, M., Jăger, E., Tiziani, H. J., Design of diffractive optical elements for CO2-laser material processing, SPIE Vol.1718 Workshop on Digital Holography (1992), p. 175-180. 